\title{
Liquid Extraction Surface Analysis Mass Spectrometry Method for Identifying the Presence and Severity of Nonalcoholic Fatty Liver Disease
}

\author{
Zoe Hall, Y Yajing Chu, and Julian L. Griffin* \\ Department of Biochemistry and Cambridge Systems Biology Centre, University of Cambridge, 80 Tennis Court Road, Cambridge \\ CB2 1GA, U.K.
}

\section{Supporting Information}

\begin{abstract}
The early stages of nonalcoholic fatty liver disease (NAFLD) are characterized by the accumulation of fat in the liver (steatosis). This can lead to cell injury and inflammation resulting in nonalcoholic steatohepatitis (NASH). To determine whether lipid profiling of liver tissue can identify metabolic signatures associated with disease presence and severity, we explored liquid extraction surface analysis mass spectrometry (LESA-MS) as a novel sampling tool. Using LESA-MS, lipids were extracted directly from the surface of ultrathin slices of liver tissue prior to detection by high-resolution mass spectrometry (MS). An isotopically labeled internal standard mix was incorporated into the extraction solvent to attain semiquantitative data. Data mining and multivariate

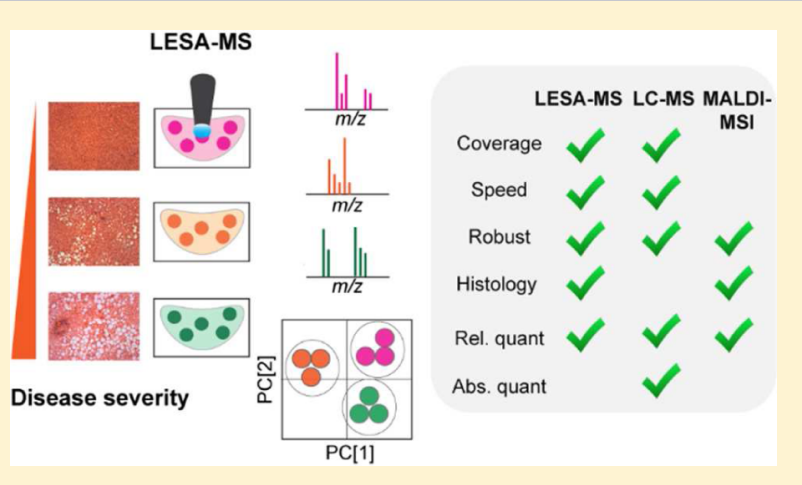
statistics were employed to evaluate the generated lipid profiles and abundances. With this approach, we were able to differentiate healthy and NAFLD liver in mouse and human tissue samples, finding several triacylglyceride (TAG) and free fatty acid (FFA) species to be significantly increased. Furthermore, LESA-MS was able to successfully differentiate between simple steatosis and more severe NASH, based on a set of short-chain TAGs and FFAs. We compared the data obtained by LESA-MS to that from liquid chromatography (LC)-MS and matrix-assisted laser desorption ionization MS. Advantages of LESA-MS include rapid analysis, minimal sample preparation, and high lipid coverage. Furthermore, since tissue slices are routinely used for diagnostics in clinical settings, LESA-MS is ideally placed to complement traditional histology. Overall LESA-MS is found to be a robust, fast, and discriminating approach for determining NAFLD presence and severity in clinical samples.
\end{abstract}

$\mathrm{N}$ onalcoholic fatty liver disease (NAFLD) is strongly associated with obesity, type 2 diabetes, and insulin resistance. ${ }^{1}$ With the global epidemic of obesity, NAFLD is becoming an increasingly important problem, particularly in Western countries where it is currently the primary cause of liver disease. ${ }^{2-4}$ NAFLD is considered to be a disease spectrum, from "simple" steatosis characterized by the accumulation of ectopic fat in the liver, to more serious conditions, including nonalcoholic steatohepatitis (NASH), cirrhosis, and hepatocarcinoma. ${ }^{5,6}$ However, it is not possible to predict disease progression on an individual basis, and so there is a great need to identify biomarkers of disease stage and to elucidate the underlying mechanisms for disease progression..$^{7-11}$

Lipidomics approaches are ideally suited for the characterization of NAFLD pathology. ${ }^{12,13}$ Increased triacylglycerides (TAG), important lipids for energy storage, are an early signature of the disease. Furthermore, oxidation of lipids containing unsaturated fatty acids is implicated in the pathogenesis of NASH, which is characterized by hepatocellular damage, inflammation, and fibrosis. ${ }^{14}$ Previously, we studied mouse and human NAFLD, using mass spectrometry imaging (MSI) and liquid chromatography-mass spectrometry (LC-
MS). ${ }^{12}$ Using MSI, we found that lipid species had specific spatial distributions within the liver which were lost with disease progression. We further established that lipid profiles, determined by LC-MS, could distinguish samples associated with NAFLD from liver samples of healthy animals. These experiments were performed on extracts from homogenized liver tissue. In this study, we sought to determine whether a novel surface sampling tool, liquid extraction surface analysis (LESA), could distinguish hepatic lipid profiles at different stages of NAFLD from ultrathin tissue slices.

LESA enables analytes to be directly sampled from tissue sections by means of a solvent droplet, which forms a liquid microjunction with the tissue surface. ${ }^{15,16}$ Analytes are dissolved in the droplet, which is then aspirated and directly infused into a mass spectrometer. Coupling LESA to MS therefore provides a detailed readout of the chemical composition of the tissue region sampled. Recent applications for LESA-MS include the analysis of fingerprints, ${ }^{17}$ detection

Received: March 24, 2017

Accepted: April 4, 2017

Published: April 4, 2017 
of specific proteins from dried blood spots and liver tissue, ${ }^{18,19}$ and distinguishing lung tumors driven by different oncogenes. ${ }^{20}$ While LESA-MS has been previously applied to biological tissue analysis, it typically provides only relative quantitation. Due to the complexity of biological samples, reporting relative changes in species between treatment groups does not always provide accurate information on whether specific analytes are significantly increased (or decreased) by a treatment or disease process. A particular challenge for quantitation is how best to incorporate an internal standard (IS) into the workflow. One way to achieve this is to include an IS mix in the extraction solvent. ${ }^{21}$ However, it is not clear whether lipid extraction via a liquid microjunction is sufficiently reproducible to enable differentiation of complex biological and clinical samples, such as those from patients at different stages of NAFLD.

Here, using mouse models of NAFLD and human clinical samples, we establish changes to the lipid profile with disease presence and severity in tissue slices. Lipids were extracted in situ from sections of liver tissue using LESA and detected using high-resolution MS. An isotopically labeled standard mix, spanning multiple lipid classes, was incorporated into the LESA extraction solvent, in order to provide semiquantitative results. Overall we found the lipid signature changes with onset and progression of NAFLD, and in particular significant increases in monounsaturated fatty acids (MUFA) and TAGs containing shorter chain fatty acids were found in NASH, compared to simple steatosis. We further compare LESA-MS to LC-MS and matrix-assisted laser desorption ionization (MALDI)-MSI and highlight several advantages of LESA-MS over traditional methods.

\section{MATERIALS AND METHODS}

Animal Study. Five-week old male $o b / o b(N=6)$ and wildtype (WT, C57BL6/J strain, $N=6$ ) mice were fed either a regular chow (RC) or high-fat (HF) diet for a period of 12 weeks. Dietary composition is described in Table S1 and detailed elsewhere. ${ }^{22}$ Mice were euthanized prior to a rapid dissection of liver tissue, which was snap-frozen in liquid nitrogen and stored at $-80{ }^{\circ} \mathrm{C}$. Serum was additionally collected, in order to perform liver function tests. All animal protocols were approved by the U.K. Home Office and the University of Cambridge Animal Welfare and Ethical Review Board and carried out by a personal license holder.

Human Samples. Nine human wedge biopsy samples were obtained from the Human Research Tissue Bank, at Addenbrooke's Hospital, Cambridge (Cambridgeshire 2 Research Ethics Committee, NRES 11/EE/0011). Samples were collected from living donors undergoing assessment; following protocols used in the Liver Clinic of Addenbrooke's Hospital, tissue was rapidly frozen following collection. Patients were selected with the following exclusion criteria: heavy alcohol consumption, hepatitis B and C, HIV, Wilson's disease, autoimmune hepatitis, hepatoxic medication, $\alpha$-1-antitrypsin deficiency. Samples were scored ${ }^{23}$ by a histopathologist for steatosis, ballooning, inflammation, fibrosis and diagnosed as normal $(N=3)$, simple steatosis $(N=3)$, or NASH $(N=3)$.

LESA-MS. Using a cryostat, $12 \mu \mathrm{m}$ frozen sections were prepared on glass microscope slides, for mouse and human liver samples embedded in Tissue-Tek OCT. Sections were airdried, and lipids were extracted from sections using LESA at multiple user-defined points across the tissue surface. LESA extraction solvent consisted of 1:2:4 chloroform/methanol/2propanol with $10 \mathrm{mM}$ ammonium acetate; $0.8 \mu \mathrm{L}$ of this solvent was dispensed onto tissue surface and incubated for 2.5 s. Analytes extracted from the surface were infused using a Triversa Nanomate (Advion BioSciences, Ithaca, NY), with capillary voltage $1.1 \mathrm{kV}, 0.3$ gas flow for 1 min into an LTQ Orbitrap Elite (Thermo Fisher Scientific, Hemel Hempstead, U.K.). Spectra were acquired in both positive and negative mode, from 200 to $1000 \mathrm{~m} / z$ at a resolution of 60000 . Lipid identity was performed by accurate mass using in-house software and confirmed using the Lipid Maps database. ${ }^{24}$ The predominant fatty acid composition was determined for selected lipids by tandem MS using collision-induced dissociation (CID) in the linear ion trap with normalized collision energy of $35 \%$.

For semiquantitative experiments, the LESA extraction solvent consisted of $25 \mu \mathrm{g} / \mathrm{mL}$ IS mix in 1:2:4 chloroform/ methanol/2-propanol with $10 \mathrm{mM}$ ammonium acetate. The mix included the following: $N$-palmitoyl- $d_{31}$-D-erythro-sphingosine (C16- $d_{31}$ ceramide), pentadecanoic- $d_{29}$ acid (15:0- $d_{29}$ FFA), heptadecanoic- $d_{33}$ acid (17:0- $d_{33}$ FFA), eicosanoic- $d_{39}$ acid $\left(20: 0-d_{39}\right.$ FFA), tetradecylphosphatidylcholine- $d_{42}$ (14:0$d_{29}$ LPC- $\left.d_{13}\right)$, 1-palmitoyl $\left(d_{31}\right)$-2-oleyl-sn-glycero-3-phosphatidylcholine (16:0- $\left.d_{31}-18: 1 \quad \mathrm{PC}\right)$, 1-palmitoyl $\left(d_{31}\right)$-2-oleyl-snglycero-3-phosphoethanolamine $\left(16: 0-d_{31}-18: 1 \mathrm{PE}\right), 1$-palmitoyl- $d_{31}-2$-oleoyl-sn-glycero-3-[phospho-rac-(1-glycerol)] (sodium salt) (16:0- $\left.d_{31}-18: 1 \mathrm{PG}\right), 1$-palmitoyl- $d_{31}-2$-oleoyl-snglycero-3-phosphoinositol (ammonium salt) (16:0- $d_{31}-18: 1$ PI), $N$-palmitoyl $\left(d_{31}\right)$-D-erythro-sphingosylphosphorylcholine $\left(16: 0-d_{31} S M\right)$, glyceryl tri(pentadecanoate- $\left.d_{29}\right) \quad\left(45: 0-d_{87}\right.$ TAG), glyceryl-tri(hexadecanoate- $\left.d_{31}\right)\left(48: 0-d_{93}\right.$ TAG) (Avanti Polar Lipids Inc., U.S.A.).

LC-MS. Lipids were extracted using the Folch method. ${ }^{25}$ An amount of $20 \mathrm{mg}$ of liver tissue was homogenized in $400 \mu \mathrm{L}$ of deionized water using a TissueLyser (Qiagen Ltd., Manchester, U.K.). Chloroform/methanol $(2: 1,1 \mathrm{~mL})$ was added, and the samples thoroughly mixed. The layers were separated by centrifugation $(12000 \mathrm{~g}, 10 \mathrm{~min})$. The extraction was performed in duplicate, with resulting organic extracts combined, dried under nitrogen, reconstituted in chloroform/ methanol $(2: 1,300 \mu \mathrm{L})$, and diluted 1:50 in 2-propanol/ acetonitrile/water (2:1:1). Samples were analyzed by LC-MS using an Accela Autosampler coupled to a LTQ Orbitrap Elite (Thermo Fisher Scientific, Hemel Hempstead, U.K.). Sample $(5 \mu \mathrm{L}$ ) was injected onto an Acuity $\mathrm{C} 18 \mathrm{BEH}$ column (Waters Ltd., Warrington, U.K.; $50 \times 2.1 \mathrm{~mm}, 1.7 \mu \mathrm{m})$ with a column temperature of $55{ }^{\circ} \mathrm{C}$. Mobile phase A was acetonitrile/water 60:40, and mobile phase B was 2-propanol/acetonitrile 90:10, both of which had $10 \mathrm{mM}$ ammonium acetate (negative ion mode), or $10 \mathrm{mM}$ ammonium formate (positive ion mode) added. A gradient run was used (Table 1 ) at a flow rate of 0.5 $\mathrm{mL} / \mathrm{min}$. The heated electrospray ionization source was at 375 ${ }^{\circ} \mathrm{C}$, the desolvation temperature was $380{ }^{\circ} \mathrm{C}$, and desolvation gas flow 40 arbitrary units. Spectra were acquired in positive and negative ion mode in the range of $200-1000 \mathrm{~m} / \mathrm{z}$ at 60000 resolution.

MALDI-MSI. Matrix solutions (10 mg/mL) of 2,5dihydroxybenzoic acid (DHB; Sigma-Aldrich, St. Louis, MO; positive ion mode) or 2,4,6-trihydroxyacetophenone (THAP; Sigma-Aldrich; negative ion mode) in 85:15 methanol/water $(\mathrm{v} / \mathrm{v})$ with $0.1 \%$ trifluoroacetic acid were administered to the tissue surface using a Suncollect MALDI spotter (KR Analytical Ltd., Cheshire, U.K.). Whole tissue slice imaging experiments were carried out using a MALDI LTQ Orbitrap XL (Thermo Fisher Scientific, Hemel Hempstead, U.K.) at $50 \mu \mathrm{m}$ step 
Table 1. Chromatography Gradient Composition for Lipidomics Open Profiling by LC-MS

$\begin{array}{ccc}\text { time }(\mathrm{min}) & \text { mobile phase A (\%) } & \text { mobile phase B (\%) } \\ 0 & 60 & 40 \\ 0.8 & 57 & 43 \\ 0.9 & 50 & 50 \\ 4.8 & 46 & 54 \\ 4.9 & 30 & 70 \\ 5.8 & 19 & 81 \\ 8 & 1 & 99 \\ 8.5 & 1 & 99 \\ 8.6 & 60 & 40 \\ 10 & 60 & 40\end{array}$

increments. Spectra were acquired in positive and negative ion mode, from 200 to $1000 \mathrm{~m} / z$ at 60000 resolution.

Data Processing. Data from LESA-MS and LC-MS experiments were converted to mzML format, and data from MALDI-MSI converted to imzML format for processing. ${ }^{26}$ Using an in-house $\mathrm{R}$ script, ions were retained above a threshold intensity and the mass-to-charge $(\mathrm{m} / z)$ ratio of ions detected were summed across integral regions of $5 \mathrm{ppm}$ to allow for alterations in $m / z$ mass accuracy across the experiment ("binning"). Lipid identification by accurate mass is achieved by searching against a library of lipid $\mathrm{m} / z$ ratios computed for all combinations of common fatty acids, lipid head-groups, and anticipated adducts in each ionization mode. Possible adducts in LESA-MS and LC-MS were $[\mathrm{M}+\mathrm{H}]^{+}$, $[\mathrm{M}+\mathrm{Na}]^{+},\left[\mathrm{M}+\mathrm{NH}_{4}\right]^{+}$in positive ion mode and $[\mathrm{M}-\mathrm{H}]^{-}$, $[\mathrm{M}+\mathrm{OAc}]^{-}$in negative ion mode. Possible adducts in MALDI-MSI were $[\mathrm{M}+\mathrm{H}]^{+},[\mathrm{M}+\mathrm{Na}]^{+},[\mathrm{M}+\mathrm{K}]^{+}$in positive ion mode, and $[\mathrm{M}-\mathrm{H}]^{-},[\mathrm{M}-\mathrm{Cl}]^{-}$in negative ion mode.
Principal components analysis (PCA) and orthogonal projection to latent structures discriminant analysis (OPLSDA) models ${ }^{27,28}$ were constructed using SIMCA 14 (Umetrics, Sweden), following normalization to the total ion count, and Pareto scaling. ${ }^{29}$ Semiquantitative experiments yielded analyte/ IS intensity ratios; group means were compared using ANOVA with Bonferroni correction for multiple comparisons.

\section{RESULTS AND DISCUSSION}

In Situ Lipid Profiling of Liver Tissue Using LESA-MS. WT and $a b / o b$ mice were fed either a regular chow (RC) or high-fat (HF) diet in order to induce NAFLD. Ob/ob mice are a commonly used genetic model in diabetes and obesity research. ${ }^{30,31}$ These mice are unable to produce the satiety hormone leptin, and consequently develop obesity and fatty liver. ${ }^{32}$ WT mice develop NAFLD when maintained on a HF diet, $^{31}$ while WT mice on a RC diet remained lean, and were used as controls. Body weights and steatosis in the liver increased in the order WT-RC $<\mathrm{WT}-\mathrm{HF}<o b / o b-\mathrm{RC} \approx o b / o b$ HF. Elevated liver enzymes (alkaline phosphatase, alanine transaminase, aspartate transaminase) in both $o b / o b$ groups indicate inflammation of the liver, with the highest levels noted for mice in $o b / o b$-RC group (Figure 1A).

In order to determine whether LESA-MS could be used to distinguish the above groups by their lipid profiles, lipids were extracted from the surface of tissue sections (Figure 1B). Five different points across each of the tissue sections were sampled, and spectra compared for control and NAFLD liver (Figure 1C-D). Typically, more than 500 lipids were identified from $>10$ lipid classes, including TAGs, diacylglycerides (DAG), ceramides, cholesteryl esters, phosphatidylcholines (PC), phosphatidylinositols (PI), phosphatidylethanolamines (PE), phosphatidylglycerols (PG), sphingomyelins (SM), and free
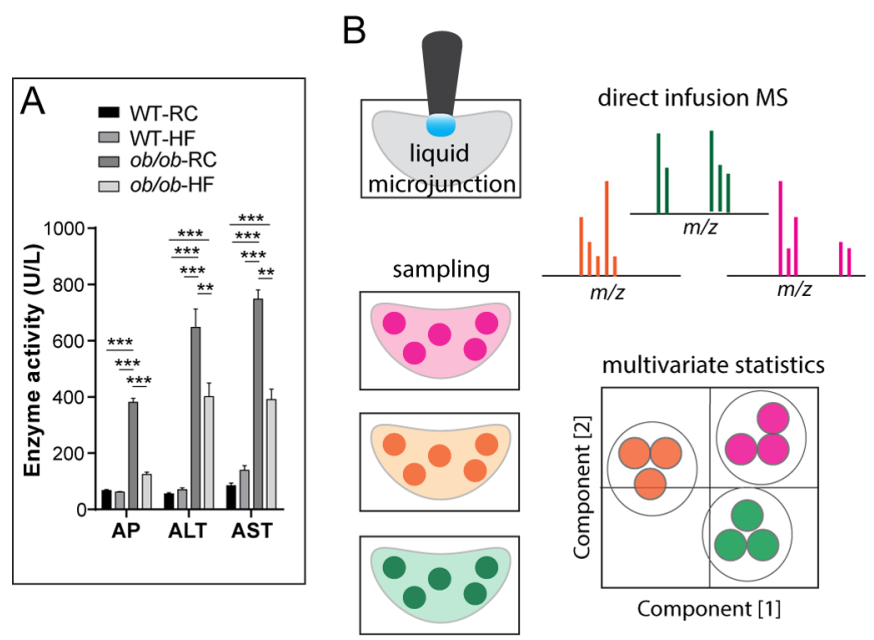
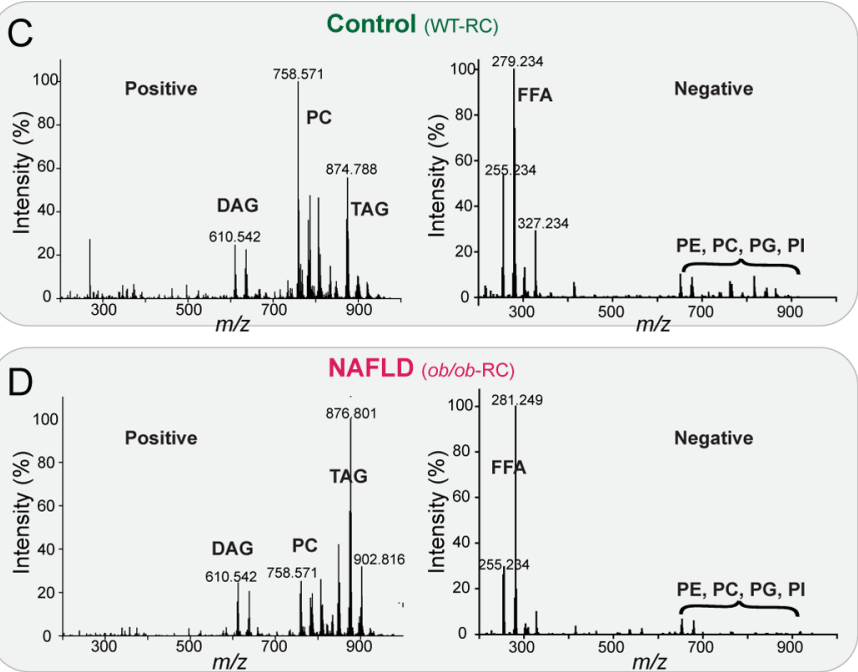

Figure 1. Liquid extraction surface analysis (LESA)-MS enables in situ sampling of fatty liver tissue. Wild-type (WT) and $o b / o b$ mice were fed a regular chow (RC) or high-fat (HF) diet. Serum alkaline phosphatase (AP), alanine transaminase (ALT), and aspartate transaminase (AST) levels (indicators of liver injury and inflammation) were all elevated in $o b / o b$-RC and $o b / o b$-HF groups (A). Data show mean \pm standard error of mean (SEM); $* P<0.05, * * P<0.01, * * * P<0.001$ (ANOVA with Bonferroni correction). Tissue sections from the mouse study were prepared for analyses by LESA-MS (B). Five different points across the tissue surface were sampled; a pipette tip brings a droplet of solvent in contact with the tissue to form a liquid microjunction. Lipids are dissolved in the droplet, which is aspirated and infused into the MS. Multiple spectra from different tissue sections were compared using multivariate statistics. Example LESA-MS spectra in positive and negative ion modes for control (WT-RC; C) and NAFLD (ob/ob-RC; D) mouse liver tissue. Major lipid classes identified in positive ion mode included diacylglycerides (DAG), triacylglycerides (TAG), and phosphatidylcholines (PC). In negative ion mode, the major lipid classes identified were free fatty acids (FFA), phosphatidylethanolamines (PE), phosphatidylglycerols (PG), phosphatidylinositols (PI), and PCs. 
Table 2. Significantly Increased Lipid Species Identified by LESA-MS in Mouse and Human NAFLD

\begin{tabular}{|c|c|c|c|c|c|c|c|c|}
\hline lipid species & $m / z$ & theor $m / z$ & $\begin{array}{l}\text { theor exact } \\
\text { mass }\end{array}$ & $\begin{array}{l}\text { mass accuracy } \\
(\mathrm{ppm})\end{array}$ & fatty acid composition & $\begin{array}{l}\text { confirmed by } \\
\text { MS/MS }\end{array}$ & diagnostic ions & lipid increased $^{a}$ \\
\hline \multicolumn{9}{|c|}{ Mouse } \\
\hline $\operatorname{FFA}(18: 1)$ & 281.249 & 281.2486 & 282.2559 & 1.4 & $18: 1$ & & & $\begin{array}{l}\text { WT-HF, } o b / o b-\mathrm{RC} \\
o b / o b-\mathrm{HF}\end{array}$ \\
\hline $\operatorname{FFA}(18: 2)$ & 279.234 & 279.2330 & 280.2402 & 3.6 & $18: 2$ & & & WT-HF \\
\hline TAG $(50: 1)$ & 850.783 & 850.7858 & 832.7520 & -3.1 & $16: 0 / 16: 0 / 18: 1$ & yes & $551.5,577.5$ & $a b / a b-\mathrm{RC}$ \\
\hline $\operatorname{TAG}(50: 2)$ & 848.772 & 848.7702 & 830.7363 & 2.1 & $16: 0 / 16: 1 / 18: 1$ & yes & $\begin{array}{l}549.5,575.5 \\
577.5\end{array}$ & $o b / o b-\mathrm{RC}, o b / o b-\mathrm{HF}$ \\
\hline TAG $(52: 2)$ & 876.801 & 876.8015 & 858.7676 & -0.6 & $16: 0 / 18: 1 / 18: 1$ & yes & $577.5,603.5$ & $\begin{array}{l}\text { WT-HF, ob/ob-RC, } \\
o b / o b-\mathrm{HF}\end{array}$ \\
\hline TAG(52:3) & 874.788 & 874.7858 & 856.7520 & 2.5 & $\begin{array}{c}16: 1 / 18: 1 / 18: 1 \text { and } \\
16: 0 / 18: 1 / 18: 2\end{array}$ & yes & $\begin{array}{r}575.5,577.5 \\
601.5,603.5\end{array}$ & $\begin{array}{l}\text { WT-HF, ob/ob-RC, } \\
o b / o b-\mathrm{HF}\end{array}$ \\
\hline TAG(52:4) & 872.7698 & 872.7702 & 854.7363 & 0.5 & $\begin{array}{c}16: 0 / 18: 2 / 18: 2 \text { and } \\
16: 1 / 18: 1 / 18: 2\end{array}$ & yes & $\begin{array}{l}573.5,575.5 \\
599.5,601.5\end{array}$ & WT-HF, $o b / o b-\mathrm{HF}$ \\
\hline TAG $(54: 3)$ & 902.816 & 902.8171 & 884.7833 & -1.2 & $18: 1 / 18: 1 / 18: 1$ & yes & 603.5 & $o b / o b-\mathrm{RC}, o b / o b-\mathrm{HF}$ \\
\hline TAG $(54: 4)$ & 900.801 & 900.8015 & 882.7676 & -0.6 & $18: 1 / 18: 1 / 18: 2$ & yes & $601.5,603.5$ & WT-HF, $o b / o b$-HF \\
\hline \multicolumn{9}{|c|}{ Human } \\
\hline $\operatorname{FFA}(16: 0)$ & 255.234 & 255.2330 & 256.2402 & 3.1 & $16: 0$ & & & $\mathbf{N A S H}^{b}$ \\
\hline $\operatorname{FFA}(18: 1)$ & 281.249 & 281.2486 & 282.2559 & 2.8 & $18: 1$ & & & steatosis, NASH \\
\hline $\operatorname{FFA}(18: 2)$ & 279.232 & 279.2330 & 280.2402 & 2.5 & $18: 2$ & & & NASH \\
\hline TAG(48:1) & 822.754 & 822.7545 & 804.7207 & -1.2 & $16: 0 / 16: 0 / 16: 1$ & yes & $549.5,551.5$ & NASH \\
\hline TAG(50:1) & 850.783 & 850.7858 & 832.7520 & -3.1 & $16: 0 / 16: 0 / 18: 1$ & yes & $551.5,577.5$ & steatosis, NASH \\
\hline TAG(50:2) & 848.770 & 848.7702 & 830.7363 & -0.5 & $\begin{array}{c}16: 0 / 16: 1 / 18: 1 \text { and } \\
16: 0 / 16: 0 / 18: 2\end{array}$ & yes & $\begin{array}{l}575.5,577.5 \\
549.5,551.5\end{array}$ & steatosis, NASH \\
\hline TAG(52:2) & 876.799 & 876.8015 & 858.7676 & -3.4 & $16: 0 / 18: 1 / 18: 1$ & yes & $577.5,603.5$ & steatosis, NASH \\
\hline TAG(52:3) & 874.784 & 874.7858 & 856.7520 & -1.6 & $\begin{array}{c}16: 1 / 18: 1 / 18: 1 \text { and } \\
16: 0 / 18: 1 / 18: 2\end{array}$ & yes & $\begin{array}{l}575.5,577.5 \\
601.5,603.5\end{array}$ & steatosis, NASH \\
\hline TAG(54:3) & 902.813 & 902.8171 & 884.7833 & -4.4 & $18: 1 / 18: 1 / 18: 1$ & yes & 603.5 & steatosis \\
\hline
\end{tabular}

${ }^{a}$ Significant increase in lipid species relative to control group ("WT-RC" in mouse study; "normal" in human study). ${ }^{b} \mathrm{NASH}-$ significantly increased lipid species in "NASH" group relative to "normal"; NASH—significantly increased lipid species in "NASH" group relative to both "normal" and "steatosis" groups.

fatty acids (FFA). Major lipid classes identified in positive ion mode included ammoniated adducts of DAGs and TAGs and protonated PCs. In negative ion mode, the major lipid classes identified were deprotonated FFAs, PEs, PGs, PIs, and acetate adducts of PCs.

Notable differences were observed in the lipid profiles of control and NAFLD liver tissue. The most striking observation was the relative increase in TAGs compared to PCs in NAFLD liver (Figure 1C-D). For instance, in positive ion mode, the most abundant ion for WT-RC liver was $m / z 758.571$ $\left(\mathrm{PC}(34: 2)[\mathrm{M}+\mathrm{H}]^{+}\right.$; Figure $\left.1 \mathrm{C}\right)$. In contrast, the most prominent ion in $o b / o b$-RC liver was $m / z$ 876.801 (TAG(52:2) $\left[\mathrm{M}+\mathrm{NH}_{4}\right]^{+}$; fatty acid composition 16:0/18:1/18:1; Figure $1 \mathrm{D}$, Table 2). In negative ion mode, the most abundant ion in WT-RC and $o b / o b$-RC liver was $m / z$ 279.234 (FFA(18:2) [M $\left.-\mathrm{H}]^{-}\right)$and $m / z 281.249\left(\mathrm{FFA}(18: 1)[\mathrm{M}-\mathrm{H}]^{-}\right)$, respectively (Figure 1C-D). While relatively high-intensity $\mathrm{m} / z 327.234$ (FFA $\left.(22: 6)[\mathrm{M}-\mathrm{H}]^{-}\right)$was observed in control liver, this ion was of relatively low abundance in NAFLD.

Lipid Profiles Differentiate Healthy and Diseased Liver in a Mouse Model of NAFLD. We used the hepatic lipid profiles, determined by LESA-MS, to discriminate the four sample groups (WT-RC, WT-HF, ob/ob-RC, ob/ob-HF; $\mathrm{H} \& \mathrm{E}$ stained sections in Figure 2A). A PCA model was constructed (Figure 2B), in which the four groups can be clearly distinguished $\left(R^{2}=0.91, Q^{2}=0.95\right)$. The first principal component ( $x$-axis) differentiated the disease state (NAFLD or control), while the second principal component ( $y$-axis) separated mice on the two different diets (RC or HF). The corresponding loadings plot revealed the lipid species most important for discriminating the groups (Figure 2C). A relative increase in PCs and saturated fatty acids $[F F A(16: 0)$, FFA(18:0)] was observed in the WT-RC group, compared to the three NAFLD groups which had increased TAGs. The $o b /$ $o b$-RC group had the largest relative increase in MUFA $[\operatorname{FFA}(18: 1), \operatorname{FFA}(16: 1)]$. In contrast, the HF-fed mice had a relatively increase in lipids containing polyunsaturated fatty acids [e.g., TAG(52:4), TAG(54:4), FFA(18:2)] compared to the groups fed a RC-diet (Figure 2C, Table 2), in line with the composition of the diet (Table S1).

An OPLS-DA model was constructed to compare the WTRC group with the combined NAFLD groups (Figure 2D; $R^{2}=$ $0.80, Q^{2}=0.97$, CV-ANOVA $\left.p<0.001\right)$. The associated Splot $^{27}$ combines the modeled covariance and correlation from the OPLS-DA model in a scatter plot (Figure 2E). This enables the determination of the most important and reliable lipid species for distinguishing the two groups (right upper and left lower quadrants for control and NAFLD liver, respectively). This highlighted the relative increase in PCs and FFA(22:6) for nonfatty liver compared to NAFLD, which was characterized by relative increases in TAG and MUFA [FFA(18:1)].

Semiquantitative Experiments Reveal Significantly Increased Lipids in NAFLD. Increased TAGs are a wellknown characteristic of fatty liver disease. However, the apparent relative decrease in PCs noted in NAFLD liver compared to the control group may be due to an actual decrease in PC content, an increase in TAGs, or a combination of the two. Furthermore, the increased TAGs in NAFLD samples may give rise to increased matrix suppression compared to samples with lower concentration of TAGs. In 


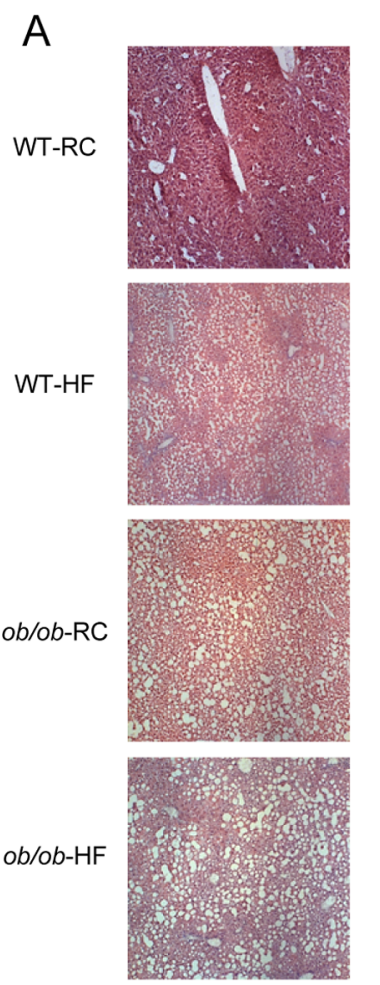

B

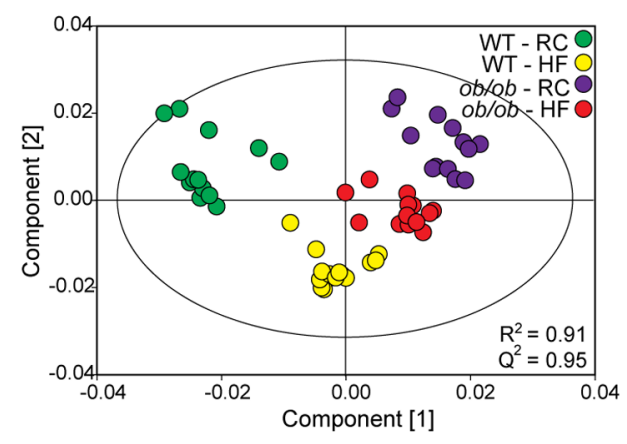

D

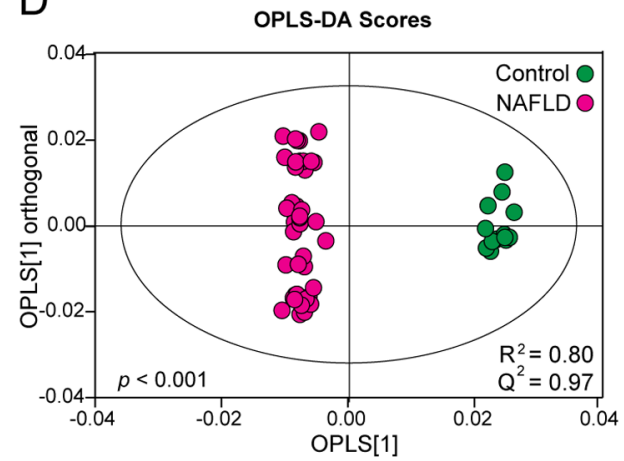

C

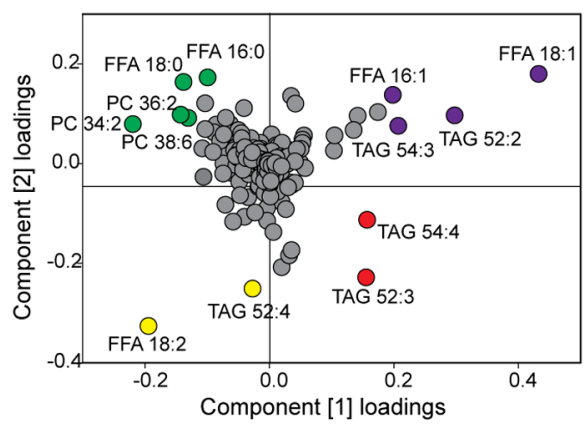

E

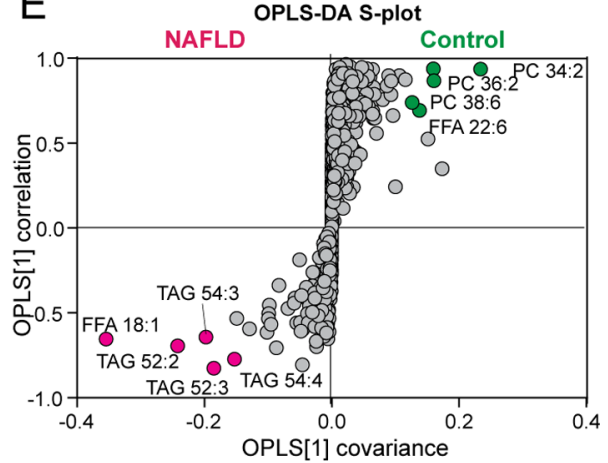

Figure 2. Lipid profile distinguishes healthy and fatty liver in the mouse. Wild-type (WT) and ob/ob mice were fed a regular chow (RC) or high-fat $(\mathrm{HF})$ diet. Liver tissue from the four groups were stained with H\&E $(\mathrm{A} ; \times 50)$, revealing increased fat accumulation (steatosis) in $o b / o b$ and $\mathrm{HF}-\mathrm{fed}$ mice, compared to control (WT-RC). LESA-MS and principal components analysis (PCA) were used to compare the hepatic lipid profiles across the four groups ( $N=3$ biological replicates per group; five repeat extractions per sample). The PCA scores (B) and corresponding loadings (C) plot reveal that the four groups can be distinguished based on their hepatic lipid profiles. An OPLS-DA model was constructed to compare control (WT$\mathrm{RC}$ ) liver with the three NAFLD groups (WT-HF, ob/ob-RC, ob/ob-HF) (D), revealing a relative increase in triacylglyceride (TAG) species and free fatty acid (FFA) 18:1 in the diseased samples compared to the control group (E).

order to address this, we prepared an IS mix, consisting of isotopically labeled compounds covering the major classes of lipids. This mix was incorporated into the LESA extraction solvent, and internal standards of the appropriate class were used to normalize peak intensities for lipids of interest.

Five areas were sampled per tissue slice, and three biological replicates analyzed per animal group. The relative standard deviation (CV) of analyte/IS peak intensity ratios for technical replicates across a single tissue slice ranged from $5 \%$ to $25 \%$, with typical values of $11 \%$ for PCs and TAGs in positive ion mode and $17 \%$ for FFAs in negative ion mode (Figure 3A-C). Group means for analytes normalized to IS were compared for those PCs, TAGs, and FFAs that were identified as important by the multivariate analysis (Figure $3 \mathrm{D}-\mathrm{F}$ ). Interestingly, there was no significant differences found between animal groups for PCs (Figure 3D). In contrast, significant differences in TAG species were noted across groups (Figure 3E, Table 2). We confirmed our previous observation that shorter chain TAGs (50-52 C), with saturated and monounsaturated acyl chains, tended to be highest in the $o b / o b$-RC group. TAGs with greater degree of unsaturation and longer chain length (52-54 C) tended to be most increased in WT-HF and $o b / o b$-HF groups. FFA(18:1) was significantly increased in all three NAFLD groups, whereas FFA(18:2) was significantly increased in the HF-fed groups only (Figure 3F, Table 2).

LESA-MS Distinguishes Severity of Human NAFLD. We applied our validated approach to thin slices of human liver tissue, which ranged from no disease (normal), to simple steatosis, and NASH (Figure 4). In addition to differentiating normal and diseased tissue, we sought to establish whether LESA-MS could be used to distinguish simple steatosis and NASH. We therefore sampled multiple points across each tissue slice from the three groups using LESA-MS and examined the generated mass spectra (Figure 4A-B). In positive ion mode, the most abundant ion for normal liver was $m / z 760.584\left(\mathrm{PC}(34: 1)[\mathrm{M}+\mathrm{H}]^{+}\right)$(Figure 4A). In contrast, NAFLD liver with either steatosis or NASH had significantly higher proportion of TAG species, with the most abundant ion $m / z$ 876.799 (TAG(52:2) $\left[\mathrm{M}+\mathrm{NH}_{4}\right]^{+}$, fatty acid composition 16:0/18:1/18:1, Table 2). In negative ion mode, the most intense ion in all three sample groups was $m / z 281.249$ $\left(\operatorname{FFA}(18: 1)[\mathrm{M}-\mathrm{H}]^{-}\right)$; however, the relative proportion of 18:1 compared to other fatty acid species appeared to increase with steatosis and NASH (Figure 4B).

We used multivariate statistics to analyze the LESA-MS spectra from each group. First, we compared normal versus NAFLD (both simple steatosis and NASH) using OPLS-DA $\left(R^{2}=0.72, Q^{2}=0.86\right.$, CV-ANOVA $p<0.001$; Figure 4C). Similar to the mouse study, normal liver tissue was characterized by a relative increase in PCs, whereas NAFLD liver had increased TAGs and MUFA. Next, we compared liver tissue with either simple steatosis or more severe NASH (Figure 4D). These two groups were well-described by an OPLS-DA model $\left(R^{2}=0.54, Q^{2}=0.79\right.$, CV-ANOVA $p<$ $0.001)$. Liver samples with NASH had a relative increase in shorter chain TAGs, with fewer double bonds (e.g., TAG(50:2); fatty acid composition 16:0/16:1/18:1 or 16:0/ 16:0/18:2, Table 2). In contrast, those with simple steatosis had 
A

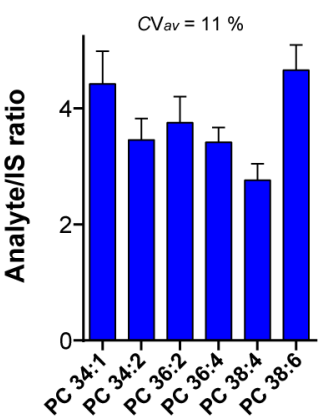

D

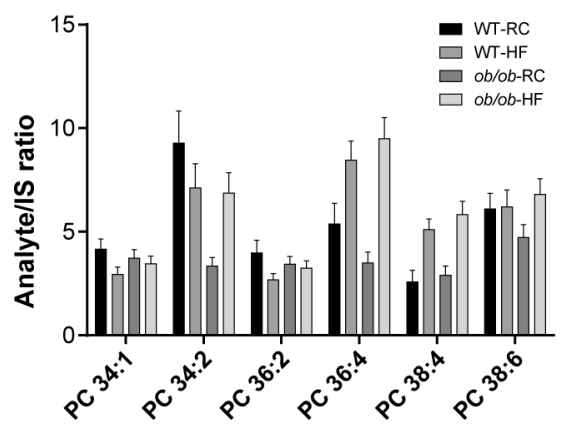

B

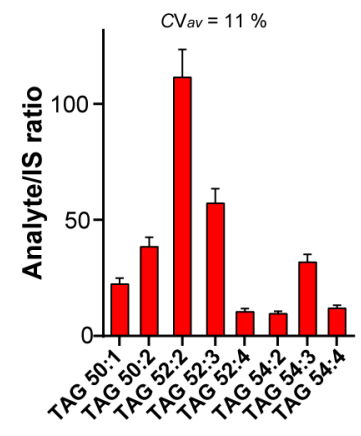

E

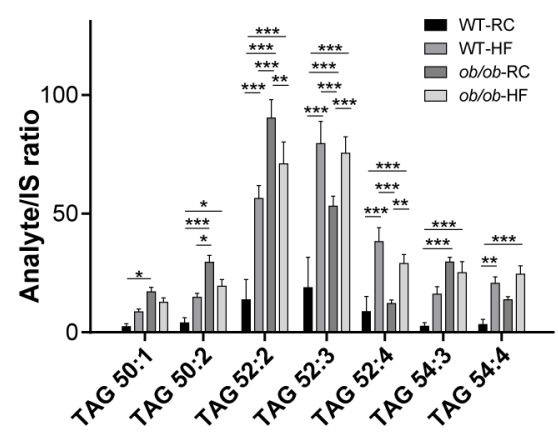

C

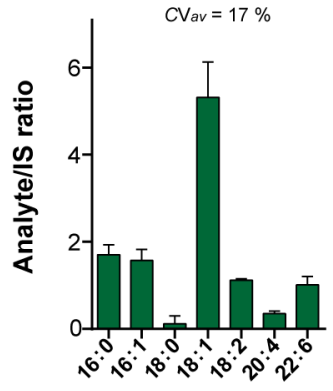

F

Figure 3. Normalization to internal standards reveal significantly changed lipids. An isotopically labeled internal standard (IS) mix was incorporated into the LESA extraction solvent, allowing semiquantitative results, by comparing analyte/IS ratios across different lipid classes (A-C). Multiple extractions per tissue slice were performed and lipids of interest measured. Typical standard deviation of key lipid species across a single fatty liver sample are shown for phosphatidylcholines (PC) (A), triacylglycerides (TAG) (B), and free fatty acids (FFA) (C). Data show mean \pm standard deviation $(\mathrm{CV})$ of five repeat extractions across tissue slice. IS-normalized lipid abundances were compared across the different groups $(N=3$ animals per group, with five repeat extractions per sample) (D-F). No significant differences were noted for PCs across the groups (D), whereas specific TAGs (E) and FFAs (F) were significantly different across groups (ANOVA with Bonferroni correction; $* P<0.05$, ** $P<0.01$, *** $P<$ $0.001)$. Data show mean \pm SEM.

increased TAGs with longer carbon chains, and a greater degree of unsaturation (e.g., TAG(54:4); fatty acid composition 18:1/ 18:1/18:2, Table 2). Furthermore, an increase in FFA(16:0) and FFA(18:1) was observed in NASH, compared to steatosis.

We next employed our semiquantitative approach to determine which of these lipids were significantly changed by NAFLD progression. We sampled five different areas per tissue slice, and three biological replicates were measured per group. Similar to the findings in the mouse study, no significant changes in PCs were found across patient groups (Figure 4E). As anticipated, significant increases in TAG species and FFAs were noted for samples with simple steatosis, and NASH compared to normal controls (Figure 4E, Table 2). Of particular note are those species which differentiate steatosis and NASH [TAG(50:1), TAG(50:2), TAG(52:2), TAG(52:3), $\operatorname{FFA}(18: 1), \operatorname{FFA}(18: 2)$, and $\operatorname{FFA}(16: 0)]$.

How Does LESA-MS Compare to Traditional Tools? We compared the data obtained from LESA-MS (Figure 5A) with traditional techniques, namely, LC-MS (Figure 5B) and MALDI-MSI (Figure 5C). While LESA-MS is a surface sampling tool, prior to LC-MS analysis lipids are extracted from homogenized liver tissue. These lipid extracts are injected onto a chromatographic column and separated by their relative affinities toward the mobile and stationery phases, followed by MS detection (Figure 5B). We analyzed tissue extracts from the WT-HF mouse group by LC-MS in both positive and negative ion mode. A similar number and identity of lipid species were detected for both LESA-MS and LC-MS.
Next, we analyzed a tissue slice from the WT-HF group using MALDI-MSI. In order to assist ionization, a matrix is first deposited onto the tissue surface. The average spectra in positive ion mode revealed predominantly TAGs, PCs, and their fragmentation products (Figure 5C), whereas negative ion mode was characterized by PIs, PEs, and FFAs. The overall coverage of lipid classes and the number of different lipid species identified was substantially lower than for LESA-MS and LC-MS, which utilize electrospray ionization (ESI). However, MALDI-MSI has excellent spatial resolution (here $50 \mu \mathrm{m}$ ), allowing the generation of images which reveal the distribution of individual lipid species across the tissue slice. For example, $\mathrm{PC}(34: 2)[\mathrm{M}+\mathrm{K}]^{+}$is shown to be distributed in the periportal zone (the area immediately surrounding portal veins) of the liver (Figure 5C, inset). In contrast, LESA-MS has a spatial resolution equivalent to the size of the solvent droplet in contact with the sample. To assess this, we analyzed a tissue slice by LESA. We subsequently coated this in matrix and reanalyzed the tissue section by MALDI-MSI (Figure 5D). The resulting ion image suggested a droplet diameter of 1.5-2 $\mathrm{mm}$-larger than zonal differences of lipids across the liver slice.

Next, we analyzed three samples from each of the four mouse groups using LC-MS, following lipid extraction from homogenized tissue. We performed PCA on the resulting lipid profiles to construct a model which accurately classified samples according to their group (Figure 5E). The first principal component separated the WT-RC group from the three NAFLD groups, whereas diet was the main factor in the 

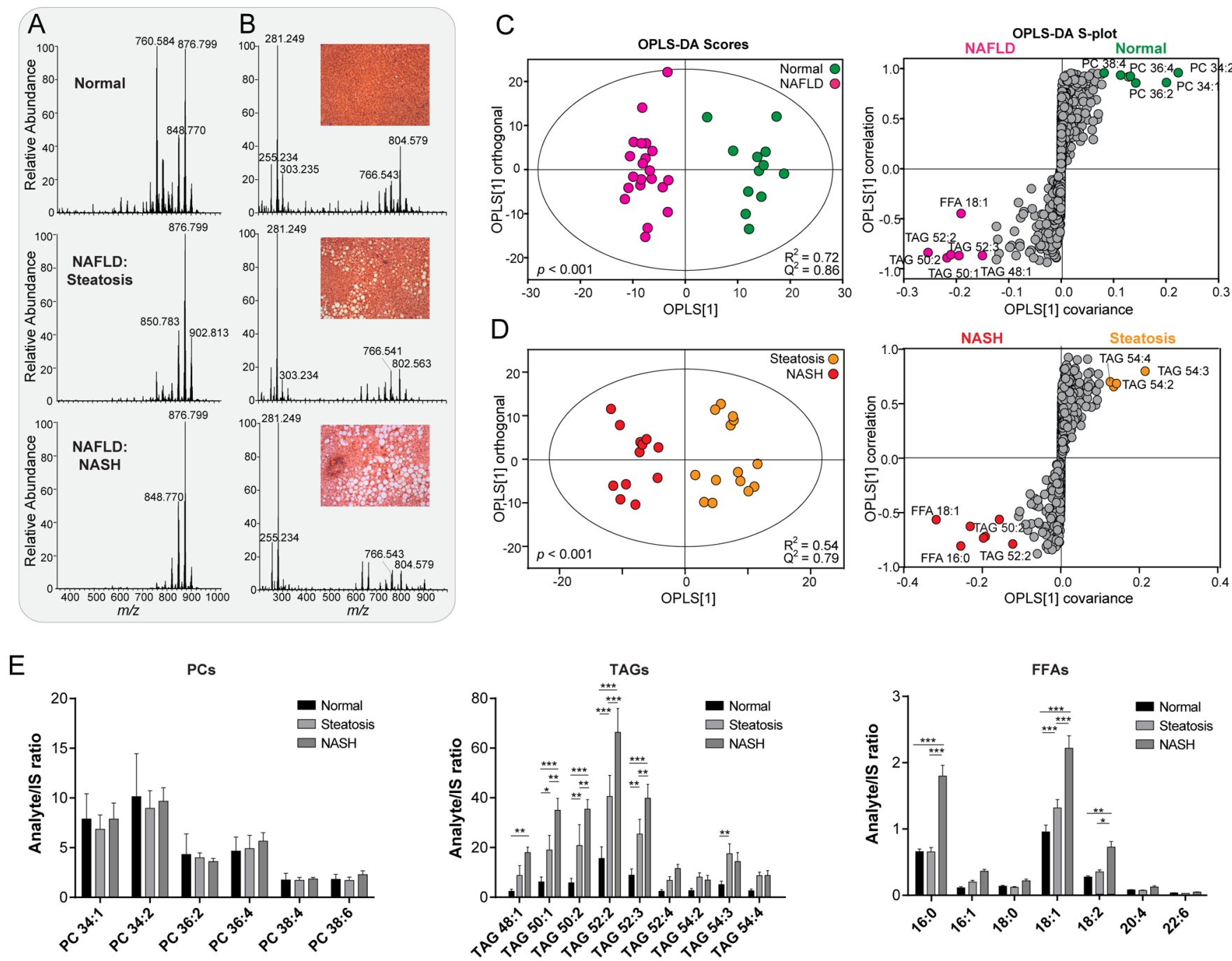

Figure 4. LESA-MS-determined presence and severity of NAFLD in human biopsies. Human liver tissue samples were analyzed by LESA-MS. Example spectra in positive (A) and negative (B) ion mode are shown for normal, simple steatosis, and NASH liver, with H\&E stained tissue $(\times 100)$ inset. An OPLS-DA model was constructed to compare normal vs NAFLD (both simple steatosis and NASH) liver (C). Similar to the mouse study, a relative increase in triacylglycerides (TAG) and free fatty acid (FFA) 18:1 in NAFLD was noted, compared to a relative increase in phosphatidylcholines (PC) in normal liver. Next, we used the LESA-MS-determined lipid profiles to discriminate between disease stage. Hepatic lipid profiles in samples with simple steatosis or NASH were compared using OPLS-DA (D). This revealed a relative increase in short-chain TAG species, FFA(16:0) and FFA(18:1), in NASH, compared to simple steatosis, which had a relative increase in longer chain TAGs. Using an internal standard (IS) mix in the extraction solvent, analyte/IS ratios were measured across groups $(N=3$ biological replicates per group, with five repeat extractions per sample) (E). While PCs were not significantly different between groups, there were significant differences noted between groups for TAGs and FFAs, with a particular increase in TAGs containing short-chain fatty acids, FFA(16:0) and (18:1), in NASH. Data show mean \pm SEM (ANOVA with Bonferroni correction; $* P<0.05, * * P<0.01$, *** $P<0.001$ ).

second principal component. Model goodness-of-fit and prediction ability $\left(R^{2}\right.$ and $Q^{2}$ values, respectively) were both $>0.9$ and thus comparable to those obtained by LESA-MS (Figure 2B). The loadings plot revealed the animal groups were distinguished by similar lipid species to those identified in LESA-MS (Figure 2C; Figure 5E). For instance, in both approaches, WT-RC group are characterized by relative increases in $\mathrm{PC}(34: 2)$ and $\mathrm{PC}(36: 2), \mathrm{WT}-\mathrm{HF}$ group by relative increases in TAG(52:4) and FFA(18:2), ob/ob-RC by relative increases in TAG(52:2) and FFA(18:1), and ob/ob-HF by a relative increase in TAG(52:3) and TAG (54:4).

Similarly, three samples from each animal group were analyzed by MALDI-MSI, and the resulting lipid profiles were used to construct a PCA model (Figure 5E). While the four animal groups were accurately clustered by their lipid profiles, the model variation explained and prediction scores were lower than for the other two analytical approaches $\left(R^{2}=0.64, Q^{2}=\right.$ 0.48). The first principal component separated the two dietary groups (HF or RC), whereas the second principal component was dominated by the genotype (WT or $o b / o b$ ). The loadings plot revealed several different lipids as being important for class distinction, including $\mathrm{PI}(38: 4)$ and $\mathrm{PE}(38: 4)$ for the WT-HF group and $\mathrm{PI}(36: 4)$ and $\mathrm{PI}(34: 2)$ for WT-RC. However, there were also similarities with data from LC-MS and LESA-MS, including PC(34:2) for WT-RC; $\operatorname{FFA}(18: 1)$ for $o b / o b-R C$; TAG(52:3) for $o b / o b$-HF (Figure 2C, Figure 5E). One explanation for the lower model score, and differences in the lipids identified as particularly important for the model, could be a result of TAGs being ionized relatively poorly in MALDI 
A
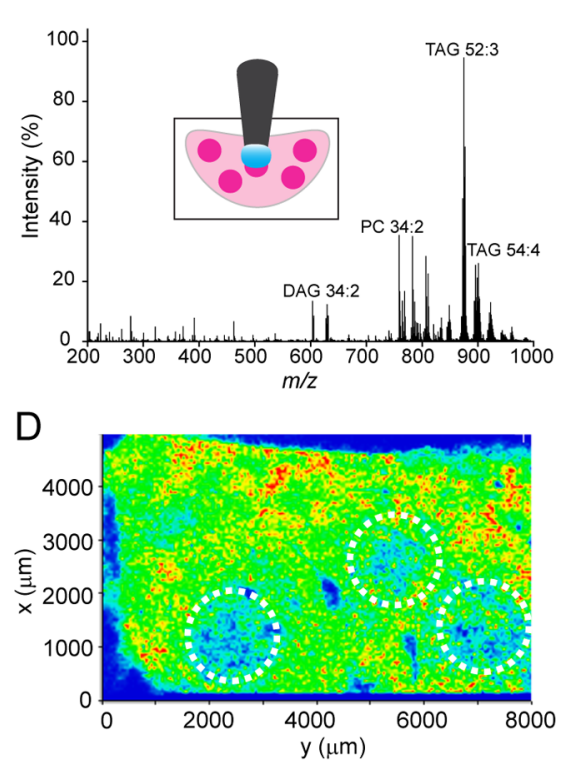

$\mathrm{F}$

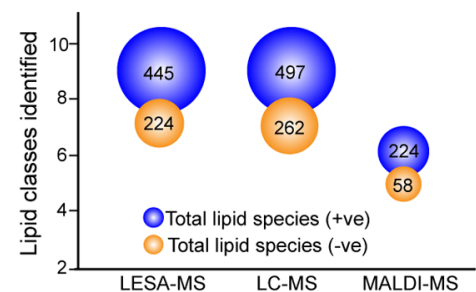

\begin{tabular}{|c|c|c|c|}
\hline & LESA-MS & LC-MS & MALDI-MS \\
\hline Coverage & 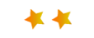 & 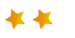 & $\star$ \\
\hline Speed & $k x$ & +4 & $X$ \\
\hline Robust & $\downarrow k$ & $\star k$ & 4 \\
\hline Spatial resolution & $\star$ & $X$ & $k \leftarrow$ \\
\hline Quantitation & 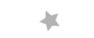 & $*$ & 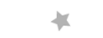 \\
\hline Sample consumption & $*$ & $\star$ & + \\
\hline
\end{tabular}

B

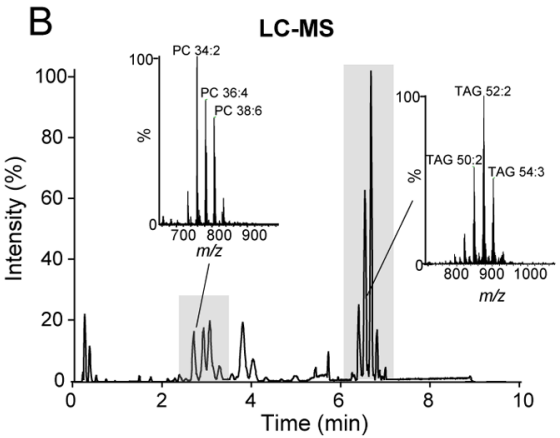

C

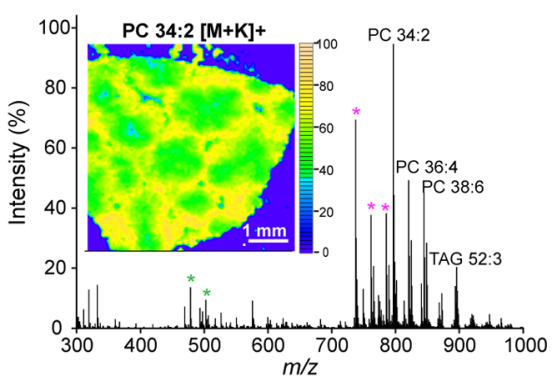

$E$
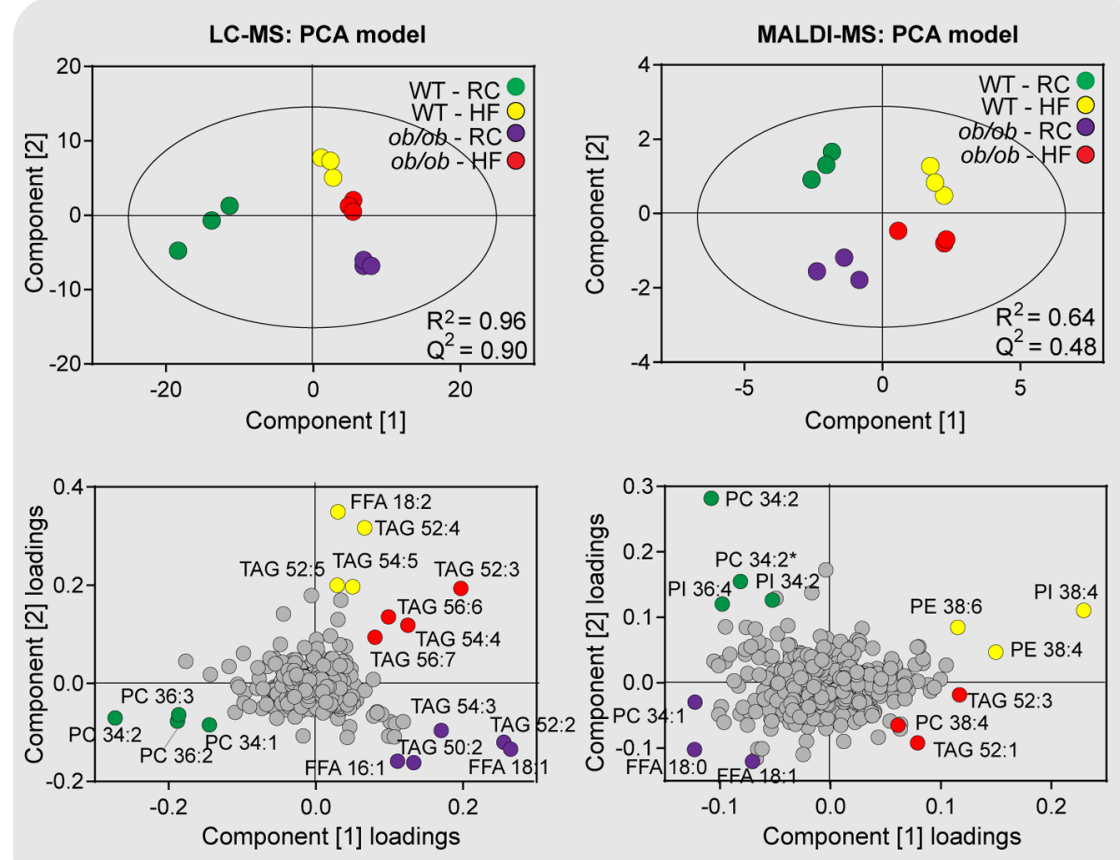

Figure 5. Comparison of LESA-MS with traditional techniques. We analyzed liver from wild-type (WT) and $o b / o b$ mice fed a high-fat (HF) or regular chow (RC) diet using LESA-MS (A), LC-MS (B), and MALDI-MSI (C). Representative positive ion spectra for WT-HF mice are shown (A-C). While both LESA-MS and MALDI-MSI were performed on thin sections of liver tissue, LC-MS analysis was performed following extraction of lipids from homogenized tissue. In LC-MS, lipids were separated by chromatography, prior to MS detection (B). Spatial distributions of lipids were observed using MALDI-MSI (C, inset), which tends to induce more fragmentation than electrospray-formed ions (LESA-MS or LCMS). Species marked with asterisks were identified as phosphatidylcholine (PC) or triacylglyceride (TAG) fragments (C). A tissue slice analyzed by LESA-MS was coated in matrix and reanalyzed using MALDI-MSI (D). Dashed white circles indicate LESA sampling regions and suggest the spatial resolution provided by this LESA method is $\sim 1.5 \mathrm{~mm}^{2}$, larger than zonal differences in lipid distributions across liver tissue. Image shows PC(38:4) $[\mathrm{M}+\mathrm{K}]^{+}$abundance (red and blue indicate highest and lowest ion intensities, respectively). Three samples per mouse group (WT-RC, WT-HF, ob/ $o b$-RC, $o b / o b$-HF) were analyzed by LC-MS or MALDI-MSI (E). Lipid profiles from the respective techniques were used to construct PCA models. Corresponding loadings plots reveal the lipids driving the differentiation of the four groups. The typical number of lipids and lipid classes identified were similar in LC-MS and LESA-MS, but greater than those identified using MALDI-MSI (F). Overall, the main advantages and limitations of each technique are given.

(Figure 5C) compared to ESI (Figure 5A-B: LESA-MS and LC-MS).

Overall, all techniques offer a robust approach to openprofiling lipidomics of liver tissue. While LESA-MS and MALDI-MSI can be performed on tissue slices, MALDI-MSI requires the deposition of a matrix on the tissue and LC-MS requires extensive sample preparation to extract lipids from tissue. Both LESA-MS and LC-MS provided excellent coverage of lipid classes and species (Figure 5F) and are rapid (here $1 \mathrm{~min}$ per LESA-MS spectrum and $10 \mathrm{~min}$ per LC-MS chromatographic run), whereas performing MALDIMSI on a whole tissue slice can take 30 min to several hours, depending on the size of sample to be analyzed. LC-MS, however, offers no spatial information, in contrast to MALDIMSI, where zonal differences of lipids were revealed. ${ }^{12}$ LESAMS has a spatial resolution of $1.5-2 \mathrm{~mm}$ (the size of the droplet), which can be useful when distinct histological features are sufficiently large or well-separated in space. For instance LESA-MS has been shown to be excellent for comparing lipid profiles of large tumors to adjacent tissue. ${ }^{20}$ However, in liver tissue, where distinction of tissue regions is less obvious, LESA-MS has more limited use as a spatial mapping tool. Finally, while MALDI-MSI and LESA-MS provide relative quantitation, LC-MS remains the gold standard to perform 
absolute quantitation, through the use of internal standards and calibration standard curves. Semiquantitative results can be achieved for LESA-MS, by incorporating isotopically labeled internal standards into the extraction solvent. The advantages and limitations of each technique are summarized in Figure 5F.

\section{CONCLUSION}

LESA-MS is shown to be a robust and discriminating approach for determining NAFLD presence and severity in a set of human liver biopsies. By extracting lipids directly from the surface of thin tissue sections, rather than using extracts from homogenized tissue, we can make use of limited material, particularly critical given the nature of human tissue biopsies. LESA-MS further offers the opportunity to perform rapid analyses with minimal sample preparation and acquisition time, providing a high coverage of lipid classes, and without complete consumption of the sample. Current diagnosis and grading of NAFLD requires biopsy slices to be examined by a pathologist, and therefore, LESA-MS is ideally suited to complement traditional histology, with potential for "bench-to-bedside" clinical applications.

\section{ASSOCIATED CONTENT}

\section{S Supporting Information}

The Supporting Information is available free of charge on the ACS Publications website at DOI: 10.1021/acs.analchem.7b01097.

Detailed composition of regular chow and high-fat diets for mouse study (PDF)

\section{AUTHOR INFORMATION}

\section{Corresponding Author}

*Phone: +44 01223 764922. E-mail: jlg40@cam.ac.uk.

\section{ORCID}

Zoe Hall: 0000-0002-1434-8329

\section{Notes}

The authors declare no competing financial interest.

\section{ACKNOWLEDGMENTS}

We gratefully acknowledge Michael Allison and Susan Davies (Addenbrooke's Hospital) for sourcing and scoring of appropriate human tissue. Xinzhu Wang and Andrew Murray (University of Cambridge) performed the animal study and are thanked for sharing samples. The Core Biochemical Assay Laboratory of Cambridge University Hospitals NHS Foundation Trust performed liver function tests. J.L.G. and Z.H. are funded by the Medical Research Council (Lipid Profiling and Signalling, MC UP A90 1006 and Lipid Dynamics and Regulation, MC PC 13030). The Human Research Tissue Bank is supported by the NIHR Cambridge Biomedical Research Centre.

\section{REFERENCES}

(1) Cohen, J. C.; Horton, J. D.; Hobbs, H. H. Science 2011, 332, 1519-1523.

(2) Lazo, M.; Clark, J. M. Semin. Liver Dis. 2008, 28, 339-350.

(3) Lazo, M.; Hernaez, R.; Eberhardt, M. S.; Bonekamp, S.; Kamel, I.; Guallar, E.; Koteish, A.; Brancati, F. L.; Clark, J. M. Am. J. Epidemiol. 2013, 178, 38-45.

(4) World Gastroenterology Organisation Global Guidelines 2012. http://www.worldgastroenterology.org/guidelines/global-guidelines/ nafld-nash (accessed December 2016).
(5) Yeh, M. M.; Brunt, E. M. Gastroenterology 2014, 147, 754-764.

(6) Wree, A.; Broderick, L.; Canbay, A.; Hoffman, H. M.; Feldstein, A. E. Nat. Rev. Gastroenterol. Hepatol. 2013, 10, 627-636.

(7) Puri, P.; Baillie, R. A.; Wiest, M. M.; Mirshahi, F.; Choudhury, J.; Cheung, O.; Sargeant, C.; Contos, M. J.; Sanyal, A. J. Hepatology 2007, 46, 1081-1090.

(8) Puri, P.; Wiest, M. M.; Cheung, O.; Mirshahi, F.; Sargeant, C.; Min, H. K.; Contos, M. J.; Sterling, R. K.; Fuchs, M.; Zhou, H.; Watkins, S. M.; Sanyal, A. J. Hepatology 2009, 50, 1827-1838.

(9) Oresic, M.; Hyotylainen, T.; Kotronen, A.; Gopalacharyulu, P.; Nygren, H.; Arola, J.; Castillo, S.; Mattila, I.; Hakkarainen, A.; Borra, R. J.; Honka, M. J.; Verrijken, A.; Francque, S.; Iozzo, P.; Leivonen, M.; Jaser, N.; Juuti, A.; Sorensen, T. I.; Nuutila, P.; Van Gaal, L.; YkiJarvinen, H. Diabetologia 2013, 56, 2266-2274.

(10) Loomba, R.; Quehenberger, O.; Armando, A.; Dennis, E. A. J. Lipid Res. 2015, 56, 185-192.

(11) Gorden, D. L.; Myers, D. S.; Ivanova, P. T.; Fahy, E.; Maurya, M. R.; Gupta, S.; Min, J.; Spann, N. J.; McDonald, J. G.; Kelly, S. L.; Duan, J.; Sullards, M. C.; Leiker, T. J.; Barkley, R. M.; Quehenberger, O.; Armando, A. M.; Milne, S. B.; Mathews, T. P.; Armstrong, M. D.; Li, C.; Melvin, W. V.; Clements, R. H.; Washington, M. K.; Mendonsa, A. M.; Witztum, J. L.; Guan, Z.; Glass, C. K.; Murphy, R. C.; Dennis, E. A.; Merrill, A. H., Jr.; Russell, D. W.; Subramaniam, S.; Brown, H. A. J. Lipid Res. 2015, 56, 722-736.

(12) Hall, Z.; Bond, N.; Ashmore, T.; Sanders, F.; Ament, Z.; Wang, X.; Murray, A. J.; Bellafante, E.; Virtue, S.; Vidal-Puig, A.; Allison, M.; Davies, S. E.; Koulman, A.; Vacca, M.; Griffin, J. L. Hepatology 2017, $65,1165-1180$.

(13) Sanders, F. W. B.; Griffin, J. L. Biol. Rev. 2016, 91, 452-468.

(14) Day, C. P.; James, O. F. W. Gastroenterology 1998, 114, 842845.

(15) Van Berkel, G. J.; Kertesz, V.; Koeplinger, K. A.; Vavrek, M.; Kong, A.-N. T. J. Mass Spectrom. 2008, 43, 500-508.

(16) Stegemann, C.; Drozdov, I.; Shalhoub, J.; Humphries, J.; Ladroue, C.; Didangelos, A.; Baumert, M.; Allen, M.; Davies, A. H.; Monaco, C.; Smith, A.; Xu, Q.; Mayr, M. Circ.: Cardiovasc. Genet. 2011, 4, 232-242.

(17) Bailey, M. J.; Randall, E. C.; Costa, C.; Salter, T. L.; Race, A. M.; de Puit, M.; Koeberg, M.; Baumert, M.; Bunch, J. Anal. Methods 2016, 8, 3373-3382.

(18) Sarsby, J.; Martin, N. J.; Lalor, P. F.; Bunch, J.; Cooper, H. J. J. Am. Soc. Mass Spectrom. 2014, 25, 1953-1961.

(19) Martin, N. J.; Bunch, J.; Cooper, H. J. J. Am. Soc. Mass Spectrom. 2013, 24, 1242-1249.

(20) Hall, Z.; Ament, Z.; Wilson, C. H.; Burkhart, D. L.; Ashmore, T.; Koulman, A.; Littlewood, T.; Evan, G. I.; Griffin, J. L. Cancer Res. 2016, 76, 4608-4618.

(21) Almeida, R.; Berzina, Z.; Arnspang, E. C.; Baumgart, J.; Vogt, J.; Nitsch, R.; Ejsing, C. S. Anal. Chem. 2015, 87, 1749-1756.

(22) Wang, X.; West, J. A.; Murray, A. J.; Griffin, J. L. J. Proteome Res. 2015, 14, 2849-2862.

(23) Bedossa, P.; Poitou, C.; Veyrie, N.; Bouillot, J.-L.; Basdevant, A.; Paradis, V.; Tordjman, J.; Clement, K. Hepatology 2012, 56, 17511759.

(24) Sud, M.; Fahy, E.; Cotter, D.; Brown, A.; Dennis, E. A.; Glass, C. K.; Merrill, A. H.; Murphy, R. C.; Raetz, C. R. H.; Russell, D. W.; Subramaniam, S. Nucleic Acids Res. 2007, 35, D527-D532.

(25) Folch, J.; Lees, M.; Stanley, G. H. S. J. Biol. Chem. 1957, 226, 497-509.

(26) Rompp, A.; Schramm, T.; Hester, A.; Klinkert, I.; Both, J. P.; Heeren, R. M.; Stockli, M.; Spengler, B. Methods Mol. Biol. 2011, 696, 205-224.

(27) Wiklund, S.; Johansson, E.; Sjöström, L.; Mellerowicz, E. J.; Edlund, U.; Shockcor, J. P.; Gottfries, J.; Moritz, T.; Trygg, J. Anal. Chem. 2008, 80, 115-122.

(28) Trygg, J.; Wold, S. J. Chemom. 2002, 16, 119-128.

(29) van den Berg, R. A.; Hoefsloot, H. C.; Westerhuis, J. A.; Smilde, A. K.; van der Werf, M. J. BMC Genomics 2006, 7, 142. 
(30) Nilsson, C.; Raun, K.; Yan, F. F.; Larsen, M. O.; TangChristensen, M. Acta Pharmacol. Sin. 2012, 33, 173-181.

(31) Takahashi, Y.; Soejima, Y.; Fukusato, T. World J. Gastroenterol. 2012, 18, 2300-2308.

(32) Perfield, J. W., 2nd; Ortinau, L. C.; Pickering, R. T.; Ruebel, M. L.; Meers, G. M.; Rector, R. S. J. Obes. 2013, 2013, 296537. 